\title{
A Variable-Included-Angle Plane-Grating-Monochromator on an Undulator for Spectroscopy and Microscopy at the Advanced Light Source*
}

\author{
Tony Warwick, Daniela Cambie, Howard A. Padmore and Malcolm R. Howells \\ Advanced Light Source, Lawrence Berkeley National Laboratory, \\ Berkeley, California 94720, USA
}

\begin{abstract}
A new beam line (beam line 11.0) at the Advanced Light Source has been designed to employ a Variable-Included-Angle Plane-Grating-Monochromator. The extended range available from a specific grating is exploited to cover energies from the carbon 1s absorption edge to the oxygen 1s absorption edge with two different gratings (1501/mm and $12001 / \mathrm{mm}$ ) with dispersion different by a factor of three. The phase space of a zone plate microscope can then be efficiently filled either at low or high resolution $(\mathrm{R}=2500$ and $\mathrm{R}=7500)$.

At the same time the monochromator is designed to cover the energy range from $75 \mathrm{eV}$ to $1500 \mathrm{eV}$ using the same two gratings at high resolution for spectroscopy. A deformable mirror pair will re-focus to a monochromatic spot $5 \times 10$ microns, matched into the acceptance of a fluorescence spectrometer.

The monochromator will operate in vertically collimated light and the free choice of focussing parameter provides a guarantee of high resolution even when the power loading is high. However, we will provide a thermally engineered pre-mirror so that the high resolution requirement at low photon energy can be met without loss of efficiency.
\end{abstract}

Keywords: monochromator, soft x-rays, microscope, dispersion

*Presented at the $7^{\text {th }}$ International Conference on Synchrotron Radiation Instrumentation, Berlin, August 21-25 2000. 


\section{General beam line details}

A beam line of this type (see Figure1) will include a collimating or focusing mirror as its first optical element. Typically, this will be a sagitally focussing, horizontally deflecting, cooled mirror. The highest required resolving power of this monochromator is $R=7500$ and the sagittal slope tolerance of the first mirror, and of the sagittal focus mirror after the grating, is about $40 \mu \mathrm{rad}$ rms. This will be an entrance slit-less beam line, the light is collimated by the first mirror and introduced as parallel light (in the vertical direction) into the vertically dispersing monochromator [1]. There is a free choice of the monochromator focusing parameter:

$$
\mathrm{Cff}=\cos \beta / \cos \alpha
$$

This choice can reduce the effect of optical slope errors at the expense of reduced efficiency.

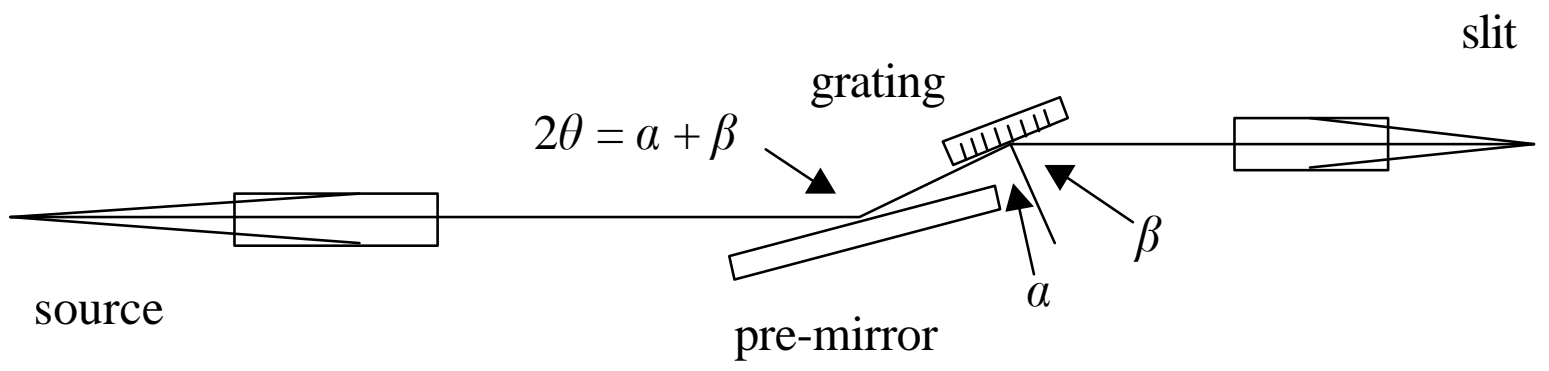

Figure 1.

Schematic diagram of an entrance slit-less, collimated, variable-included-angle, planegrating- monochromator.

\section{Power load on the pre-mirror}

One point of this paper is to correctly compute the power load on the monochromator pre-mirror as the photon energy is tuned and to compare thermal effects under varying operating conditions. We model the undulator and monochromator tuning together, with the selected photon energy being produced with peak brightness at the fundamental frequency of the undulator emission. The computed harmonic power density on axis for each harmonic is scaled according to the Henke reflectivity of the optics [2] (at the appropriate energy and angle of incidence) and the absorbed or reflected power density on-axis is computed as the sum over odd harmonics. This sum is used to scale the angular distribution of total power to approximate the angular distribution of power absorbed. All computations are made for the ALS at $1.9 \mathrm{Gev}$ with $400 \mathrm{~mA}$ of stored electrons. Our $5 \mathrm{~cm}$ period elliptical undulator with 37 periods produces $1260 \mathrm{Watts}$ total power with the fundamental tuned to the lowest energy $(75 \mathrm{eV})$. The monochromator optics are farthest from grazing incidence here so the reflectivity for high energy photons is less, and the spreading of the heat along the optical surface is at its minimum. Therefore, there is a strong increase of absorbed power density as the selected photon energy is reduced. 


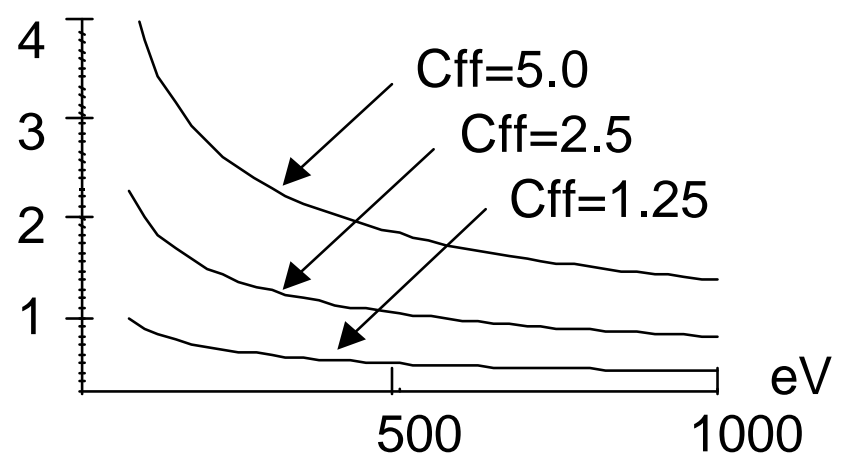

Figure 2.

The rms slope error ( $\mu \mathrm{rad})$ of the pre-mirror corresponding to a resolving power $\mathrm{R}=7500$ (FWHM) from the $1501 / \mathrm{mm}$ grating.

The tangential slope error tolerances for the plane pre-mirror and for the grating depend on the angles of the optics and become critically tight at angles far from grazing incidence (see Figure2). The pre-mirror power loading is much higher than that of the grating, so it is on this optic that thermal deformations might limit the monochromator resolution. For example, in the most difficult operational situation on this beam line $(75 \mathrm{eV}$, $\mathrm{Cff}=1.25,1501 / \mathrm{mm}$ ) the pre-mirror absorbs $0.2 \mathrm{Watts} . \mathrm{mm}^{-2}$ and the pre-mirror deformation tolerance is $1.0 \mu \mathrm{rad}$ rms. The power density absorbed by the grating is ten times less.

\section{Typical design for a cooled pre-mirror}

In this study a typical water cooled substrate for the pre-mirror has been considered. The geometry of the mirror is depicted in Figure 3.

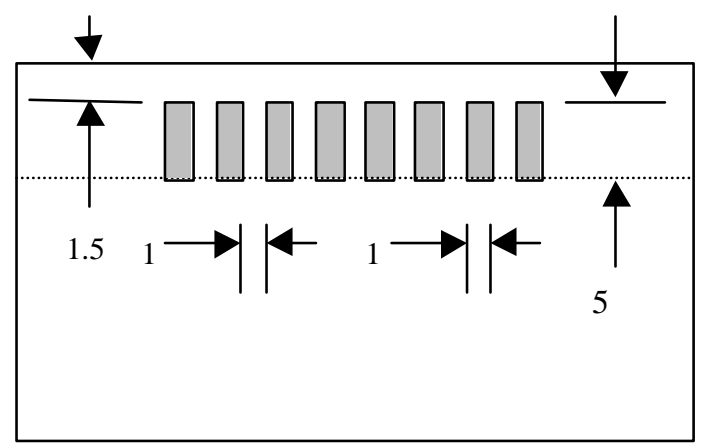

Figure 3.

Typical pre-mirror cross section with water channels. The width is immaterial. A mirror like this is easily manufactured in frit-bonded silicon or brazed copper.

The cooling channels are cut along the length of a bonded face plate. This design is possible both for silicon mirrors, using a frit bonding technique [3] and for brazed 'glidcop' mirrors. Both techniques have been successfully implemented at the ALS. The heat is applied to a quarter FEA model as a surface load, and the model substrate is kinematically constrained. In the FEA analysis we have computed the deformations for both 'glidcop' and 
silicon mirrors with the geometry shown. Material properties [4] are assumed constant since the temperature changes in the mirrors are small. A flow rate of $\sim 0.16 \mathrm{gpm}$ in each channel leads to a velocity of $2 \mathrm{~m} / \mathrm{s}$, enough to ensure a turbulent flow and a convection coefficient of $\sim 10,000 \mathrm{~W} / \mathrm{m}^{2} \mathrm{~K}[5]$. The pressure drop in the channels has been calculated to be $8 \mathrm{psi} / \mathrm{m}$. The mirror is $450 \mathrm{~mm}$ long. Calculations have been performed to ensure that the pressure of the coolant (60 psi) does not produce significant deformations of the hot wall. Thermal deformations are shown in Figure4.
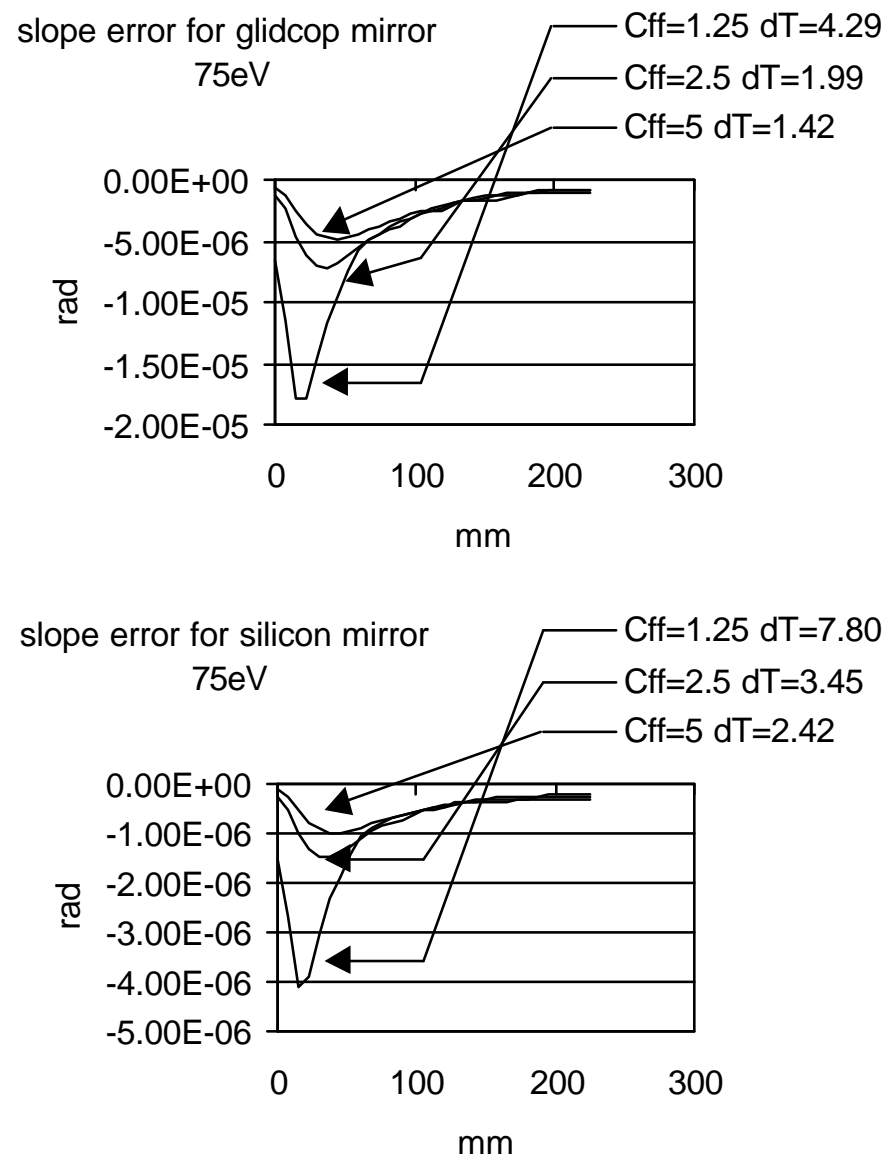
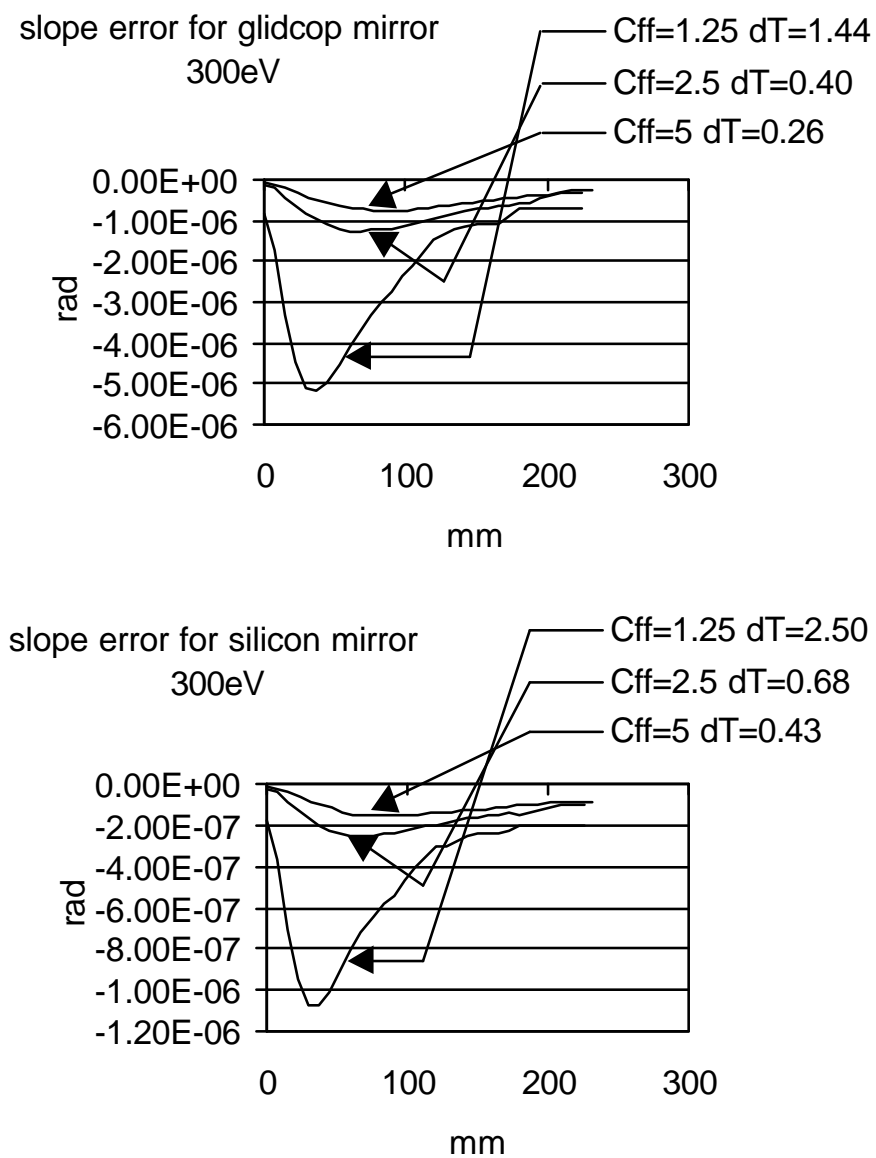

Figure 4.

The deformations (radians) and peak surface temperature rise $\left({ }^{\circ} \mathrm{C}\right)$ of the pre-mirror under the thermal load with $400 \mathrm{~mA}$ electron current, with the monochromator and undulator tuned together to $75 \mathrm{eV}$ and $300 \mathrm{eV}$, and $\mathrm{Cff}=1.25 .2 .5$ and 5.0

A silicon mirror will not limit the monochromator resolution except at low energies and low Cff. It is a viable solution. Bonding metal tubes to silicon without leaks is a challenge, but the techniques for silicon machining and silicon-to-silicon bonding in this geometry are known. A mirror in copper does not suffer from these fabrication difficulties, but its thermal deformations will limit the monochromator resolution except at higher photon energy and at large values of Cff. Typically, the diffraction efficiency will be maximized at 
$\mathrm{Cff} \simeq 1.5$. Higher values correspond to more grazing incidence angles for the optics and greatly reduced absorbed power density. Higher Cff values also reduce the sensitivity of the monochromator resolution to optical slope errors, proportional to $\cos \alpha$. The most stringent conditions are at low photon energy and low Cff. Operating at higher values of Cff can guarantee good resolution even at the lowest energy at the cost of decreased diffraction efficiency and decreased vertical aperture. Comparing $\mathrm{Cff}=1.5\left(\alpha=88.96^{\circ}\right)$ to $\mathrm{Cff}=4$ $\left(\alpha=86.39^{\circ}\right)$ in this beam line at $75 \mathrm{eV}$, the computed diffraction efficiency falls from $20 \%$ to $8 \%$ and the vertical acceptance of the grating is reduced by a factor of 3.5.

\section{Acknowledgements}

This work arose from very useful discussions Rolf Follath, Steve Hulbert, Uwe Flechsig and Christoph Quittmann.

This work was supported by the Director, Office of Energy Research, Office of Basic Energy Sciences, Materials Sciences Division of the U.S. Department of Energy, under Contract No. DE-AC03-76SF00098

\section{References}

1. K.J.S.Sawhney, F.Senf, M.Scheer, F.Schafers, J.Bahrdt, A.Gaupp and W.Gudat, Nucl. Instr. Methods. A390 (1997) 395

2. B.L.Henke, P.Lee, T.J.Tanaka, R.L.Shimabukuro and B.K.Fujikawa, Atomic and Nuclear Data Tables 27 (1982) 1

3. T.Tonnessen, In-Sync Optical Corporation.

4. M.R.Howells Opt. Eng. 35(1996)1187

5. F.P.Incropera, D.DeWitt, 'Fudamentals of Heat and Mass Transfer", John Wiley and Sons, New York, 1990 